\title{
Nocturnal desaturation in patients with stable heart failure
}

\author{
A D Staniforth, W J M Kinnear, R Starling, A J Cowley
}

\begin{abstract}
Objective-To determine the prevalence of sleep disordered breathing within a United Kingdom heart failure population. Subjects-104 patients and 21 matched normal volunteers.

Methods-Overnight home pulse oximetry with simultaneous ECG recording in the patient group; daytime sleepiness was assessed using the Epworth sleepiness scale (ESS); 41 patients underwent polysomnography to assess the validity of oximetry as a screening test for CheyneStokes respiration.
\end{abstract}

Results-Home oximetry was a good screening test for Cheyne-Stokes respiration (specificity $81 \%$, sensitivity $87 \%$ ). Patients with poorer New York Heart Association (NYHA) classes had higher sleepiness scores $(p<0.005)$. Twenty three patients had "abnormal" patterns of nocturnal desaturation suggestive of CheyneStokes respiration. The mean (SEM) frequency of dips in $\mathrm{SaO}_{2}$ exceeding $4 \%$ was $10.3(0.9)$ per hour in the patients and $4.8(0.6)$ in normal controls $(p<0.005)$. Ejection fraction correlated negatively with dip frequency $(r=-0.5 . p<0.005)$. The patient subgroup with $\geqslant 15 \mathrm{dips} / \mathrm{hour}$ had a higher mean (SEM) NYHA class (3.0 (0.2) $v 2.3(0.1), \mathrm{p}<0.05)$, and experienced more ventricular ectopy (220 (76) $v$ 78 (21) beats/hour, $p<0.05)$. There was no excess of serious arrhythmia.

Conclusions-Nocturnal desaturation is common in patients with treated heart failure. Low ejection fraction was related to dip frequency. Lack of correlation between dips and ESS suggests that arousal from sleep is more important than hypoxia in the aetiology of daytime sleepiness in heart failure. Overnight oximetry is a useful screening test for CheyneStokes respiration in patients with known heart failure.

(Heart 1998;79:394-399)

Keywords: Cheyne-Stokes respiration; nocturnal desaturation; heart failure

In 1818 Cheyne first described a case of periodic respiration during sleep in a patient with terminal heart failure. ${ }^{1}$ Cheyne-Stokes respiration is now accepted as a sign of severe left ventricular dysfunction. ${ }^{2}{ }^{3}$ Studies performed in the United States have shown that sleep apnoea is common in moderate to severe heart failure. ${ }^{4}$ The presence of Cheyne-Stokes respiration is important since it is an independ- ent marker of increased mortality, ${ }^{5}$ and because the repeated episodes of nocturnal hypoxaemia and arousal may be detrimental to sleep quality, leading to marked daytime symptomatic impairment.

The prevalence of occult sleep disordered breathing in heart failure patients in the United Kingdom is unknown. Although polysomnography is accepted as the gold standard for investigating sleep disorders, in Europe and the United Kingdom it is customary to use overnight pulse oximetry as a screening test. Oximetry has the advantage of being cheap, comfortable, and convenient for the patient. It has the drawback that, because it does not directly measure airflow, it cannot distinguish between central and obstructive apnoeas. If the clinical setting is taken into account when interpreting oximetry recordings, however, it may be that full polysomnography is unnecessary.

Our study was performed in patients with stable heart failure in their own homes. All New York Heart Association (NYHA) functional classes were represented, and all patients were receiving standard medical treatment. The purpose of the study was to validate the use of oximetry as a screening tool for CheyneStokes respiration in patients with known heart failure, to assess the prevalence and severity of nocturnal desaturation, and to compare these with the results of Holter monitoring.

\section{Methods}

SUBJECTS AND QUESTIONNAIRES

One hundred and four patients were recruited from a specialist heart failure clinic. Patients of any age, sex, aetiology, duration, or severity of symptoms were included. Subjects with known pulmonary disorder or stroke were excluded. All patients had stable cardiac failure with no change in medication for four weeks before study and no evidence of fluid retention at the time of study. Only patients receiving standard medical treatment were included in the study.

Table 1 Baseline characteristics of oximetry patients $(n=104)$

\begin{tabular}{ll}
\hline & Mean (SD) \\
\hline Symptom duration (months) & $40.2(52.6)$ \\
Alcohol intake (units/week) & $6.5(9.9)$ \\
Frusemide dose (mg) & $95.7(97.9)$ \\
Captopril dose (mg) & $95.3(65.2)$ \\
NYHA class (number of patients) & \\
I & 14 \\
II & 41 \\
III & 40 \\
IV & 9 \\
\hline
\end{tabular}

^All ACE inhibitors are expressed as equivalent doses of captopril. 


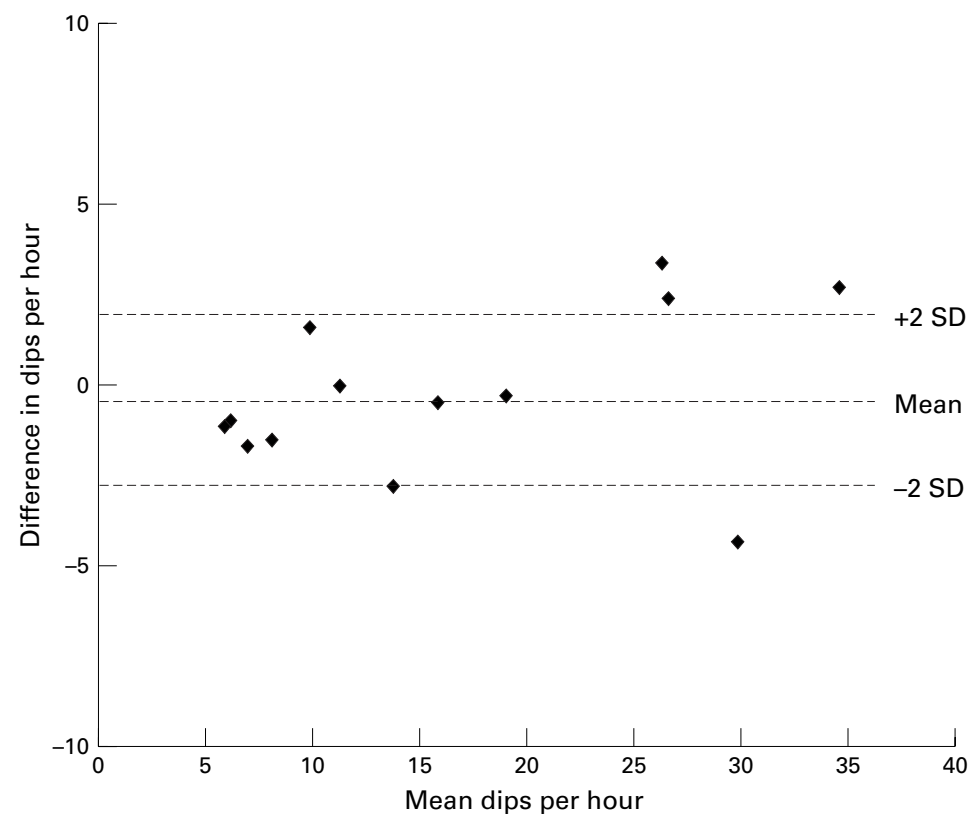

Figure 1 Bland and Altman plot showing the short term reproducibility of overnight home pulse oximetry in patients with controlled heart failure. $\zeta_{w}=1.3$ dips $/ \mathrm{h}$; mean difference $=-0.2$ dips $/ h ;$ coefficient of variation $=7.9$.

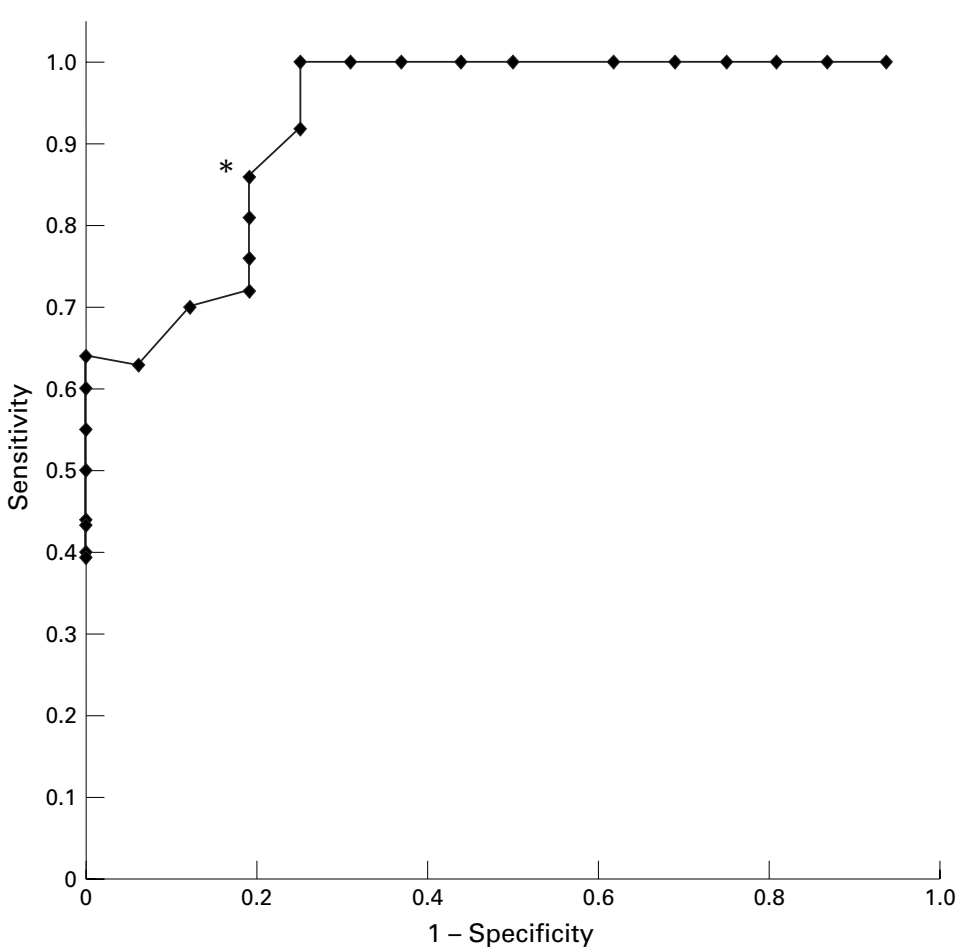

Figure 2 Receiver-operator curve demonstrating the ability of pulse oximetry to discriminate between normal breathing and Cheyne-Stokes respiration in heart failure. *Indicates a desaturation index of 15 dips/h (see text).

Table 2 Comparison of baseline characteristics and Holter analysis for patients grouped according to frequency of dips in $\mathrm{SaO}_{2} \geqslant 4 \%$

\begin{tabular}{llll}
\hline & Dips $<15 / h(n=81)$ & Dips $\geqslant 15 / h(n=23)$ & p value \\
\hline Age & $64(1.2)$ & $66.6(1.6)$ & NS \\
Body mass index $\left(\mathrm{m}^{2} / \mathrm{kg}\right)$ & $26.6(0.4)$ & $26.2(0.6)$ & $\mathrm{NS}$ \\
Ejection fraction & $0.48(0.02)$ & $0.31(0.03)$ & $<0.005$ \\
Alcohol (units/week) & $7.8(1.1)$ & $5(2.3)$ & $\mathrm{NS}$ \\
NYHA class & $2.3(0.1)$ & $3(0.2)$ & $<0.005$ \\
Epworth sleepiness scale & $7.7(0.4)$ & $7.1(0.9)$ & $\mathrm{NS}$ \\
Extrasystoles (per hour) & $78(22)$ & $220(76)$ & 0.05 \\
\hline
\end{tabular}

Standard medical treatment was defined as an angiotensin converting enzyme (ACE) inhibitor in combination with that dose of diuretic required to control signs of pulmonary or pedal oedema. Patients who were not taking ACE inhibitors owing to documented intolerance were eligible for study. Digoxin and $\beta$ blockers were not considered to be standard treatment for heart failure.

Heart failure was diagnosed on the basis of a suggestive medical history and examination findings together with visually impaired left ventricular function on cross sectional echocardiography. Left ventricular function was quantified by $M$ mode echocardiography. Only subjects with either a left ventricular diastolic diameter greater than $5.5 \mathrm{~cm}$ or an ejection fraction less than $45 \%$ were eligible for study.

Twenty one normal volunteers were also studied, matched for age, sex, and body mass index. These subjects were recruited from responders to a series of adverts placed around the hospital building. None had a history or examination findings suggestive of stroke, pulmonary, cardiovascular, or sleep disorders. Normal left ventricular function was confirmed by the presence of a normal 12 lead ECG and normal systolic function on cross sectional and $M$ mode echocardiography (see above).

All subjects completed a self administered questionnaire based on the Epworth sleepiness scale (ESS) to assess symptoms of daytime sleepiness. This test generates a score ranging from 0 to 24 (higher scores reflecting increased sleepiness). The ESS has been validated in patients with known sleep disorders, ${ }^{6}$ and correlates well with sleep latency (an EEG based measurement of sleepiness).?

\section{OXIMETRY STUDIES}

One hundred and four patients and 21 normal controls agreed to overnight home oximetry using the Biox 3700 pulse oximeter (Ohmeda, Colorado, USA). They were instructed in the operation of the oximeter and were fitted with a Holter monitor (patients only) in the afternoon of the day of their sleep study. Thirteen patients agreed to home oximetry on two successive night to allow the short term reproducibility of this technique to be assessed in heart failure. The apparatus records the lowest percentage transcutaneous oxygen concentration $\left(\mathrm{SaO}_{2}\right)$ detected over successive 12 second time intervals up to a maximum of eight hours. ${ }^{8}$ This equipment has previously been used in a large population based obstructive sleep apnoea screening study. ${ }^{9}$ The data were analysed to calculate the overnight minimum $\mathrm{SaO}_{2}$, mean $\mathrm{SaO}_{2}$, dip frequency (defined as a fall in $\mathrm{SaO}_{2}$ of $\geqslant 4 \%$ ), the percentage of oximetry sleep time spent with an $\mathrm{SaO}_{2}$ of $\leqslant 90 \%$, and the total oximetry time. All oximetry recordings were also inspected by a single observer (WJMK) blinded to the identity of the subjects. Recordings were classified as "normal" (< 4.9 desaturation events an hour), "equivocal" (5 to 14.9 desaturation events an hour), or "abnormal" (> 15 desaturation events an hour) according to the severity and pattern of nocturnal desaturation observed. 
Table 3 Results of overnight home pulse oximetry in 104 patients and 21 matched controls

\begin{tabular}{llll}
\hline & $\begin{array}{l}\text { Normal controls } \\
(n=21)\end{array}$ & $\begin{array}{l}\text { Heart failure } \\
(n=104)\end{array}$ & p value \\
\hline Age & $65.9(1.4)$ & $64.1(0.9)$ & $\mathrm{NS}$ \\
Body mass index $\left(\mathrm{kg} / \mathrm{m}^{2}\right)$ & $25.6(0.6)$ & $26.7(0.3)$ & $\mathrm{NS}$ \\
Ejection fraction & $0.68(0.03)$ & $0.41(0.02)$ & $<0.005$ \\
& & & \\
Oximetry data & $4.8(0.6)$ & $10.3(0.9)$ & $<0.005$ \\
$\begin{array}{l}\text { Dip frequency (per hour) } \\
\text { Mean SaO }_{2}(\%)\end{array}$ & $95.9(0.2)$ & $94.0(0.2)$ & $<0.005$ \\
Minimum SaO $_{2}(\%)$ & $89.6(0.9)$ & $84.5(0.6)$ & $<0.005$ \\
Oximetry time with SaO $_{2} \leqslant 90 \%(\%)$ & $0.2(0.1)$ & $5.7(1.2)$ & $<0.005$ \\
Oximetry analysis $_{\text {Normal recording }}$ & & & \\
Equivocal recording & 15 & 46 & $<0.05$ \\
Abnormal recording & 6 & 35 & \\
\hline All resuls givn & 0 & 23 & \\
\hline
\end{tabular}

All results given as mean (SEM).

$\mathrm{SaO}_{2}$, oxygen saturation by pulse oximetry.

Overnight ECG recording was performed in the patient group using a Tracker-2 Holter monitor (Hertford Medical, Hertford, UK). All tapes were analysed for the presence of ventricular arrhythmia by a single observer (ADS) using the Medilog Excel ECG analysis system (Oxford Medical, Oxford, UK). The study was approved by the hospital ethics committee.

POLYSOMNOGRAPH SUBSTUDY

Forty one of the heart failure subjects were selected at random and admitted to hospital for overnight polysomnography. The aim of the
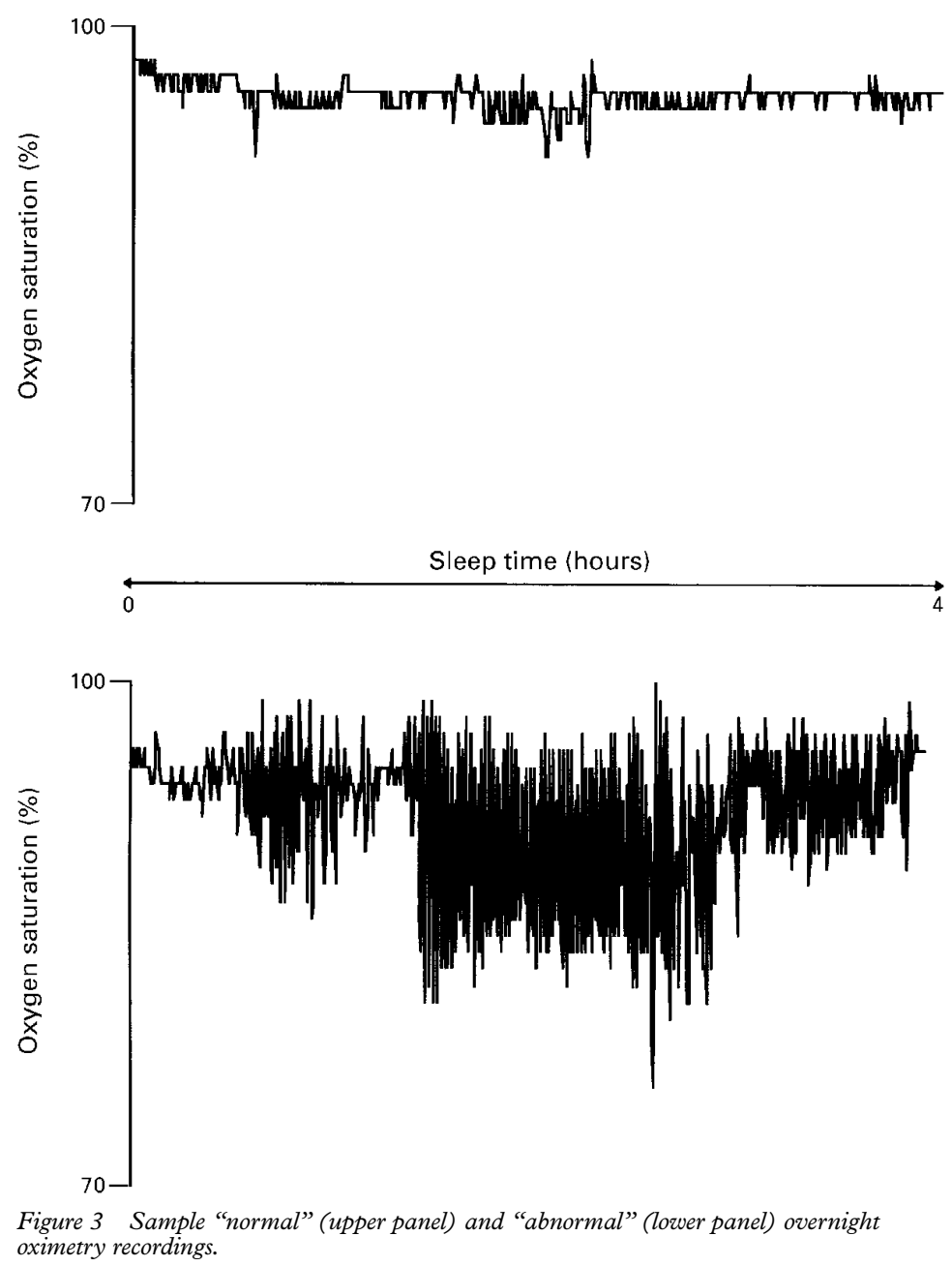

polysomnography substudy was to determine the precise origin of any episodes of nocturnal desaturation, and to validate the use of overnight pulse oximetry as a screening test for Cheyne-Stokes respiration in patients with known heart failure.

The MEDILOG multiparameter analysis system (Oxford Medical) was used to record the polysomnographs. Surface electrodes were used to record the encephalogram, electrooculogram, and mental electromyogram. Respiratory movements were recorded by impedance. ECG was recorded with standard limb leads. Arterial oxygen saturation was measured by pulse oximetry. Oral and nasal airflow were detected with thermistors attached to the upper lip and connected to the multichannel recorder. The data were analysed using the MEDILOG 9000-III replay and display system and a SS90-III sleep stager (Oxford Medical). Standard criteria were used to define abnormal sleep patterns and sleep stage. ${ }^{10}$

The following definitions were used to classify abnormal breathing patterns and arousal. Apnoea was defined as an absence of airflow for more than 10 seconds. Central apnoeas were defined by the absence of airflow for 10 seconds accompanied by an absence of chest wall movement. Hypopnoea was defined as a reduction in the amplitude of respiratory movement for more than 10 seconds to less than $50 \%$ of the maximum amplitude recorded during the preceding breathing cycle. The presence of Cheyne-Stokes respiration was defined as a central apnoea index of $\geqslant 10$ an hour, in combination with the characteristic pattern of crescendo-decrescendo hyperpnoea-central apnoea periodic breathing. Arousal was defined as awakening from sleep for more than five seconds, as evidenced by the simultaneous occurrence of $\alpha$ activity on the electroencephalogram, electromyogram activation, and eye movements. ${ }^{11}$ All computerised analyses were subsequently checked from inspection of the raw data traces by an independent EEG technician (RS).

\section{STATISTICAL ANALYSIS}

Unless otherwise stated all results are presented as means (SEM). The unpaired $t$ test and Mann-Whitney $U$ test were used to assess the level of significance between groups of numerical data. The $t$ test was only applied to normal data if the equal variance assumption was upheld on $\mathrm{F}$ test. Categorical data were analysed using the $\chi^{2}$ test. All differences were taken to be significant at $\mathrm{p}<0.05$.

\section{Results}

REPRODUCIBILITY AND VALIDATION OF OVERNIGHT HOME OXIMETRY

A Bland and Altman plot of the short term reproducibility of overnight home oximetry in patients with heart failure is shown in fig 1 . Figure 2 is the receiver-operator curve showing the ability of overnight oximetry to detect Cheyne-Stokes respiration in patients with known heart failure. Using a desaturation index of 15 dips a minute as the cut off point, 
Table 4 Results of full polysomnography in 41 subjects selected at random from heart failure patients

\begin{tabular}{lll}
\hline & No CSR & CSR \\
\hline Actual sleep time (AST) (mins) & $330(19)$ & $358(21)$ \\
Non-REM 1-2 sleep (\% of AST) & $55(3)$ & $60(3)$ \\
Non-REM 3-4 sleep (\% of AST) & $29(2)$ & $24(3)$ \\
REM sleep (\% of AST) & $16(2)$ & $14(2)$ \\
Arousal index (per hour AST) & $5.4(0.8)$ & $10(1.9)^{\star}$ \\
Desaturation index (per hour TST) & $6.3(0.9)$ & $26.4(3)^{\star \star}$ \\
Minimum Sao & $86.8(0.8)$ & $81.7(2.5)^{\star}$ \\
Apnoea/hypopnoea index (per hour AST) & $24.2(3)$ & $52.5(3.2)^{\star \star}$ \\
Central apnoea index (per hour AST) & $2(0.5)$ & $28.4(2.9)^{\star \star}$ \\
\hline
\end{tabular}

Results are given as mean (SEM)

${ }^{\star} \mathrm{p} \leqslant 0.05 ;{ }^{\star \star} \mathrm{p} \leqslant 0.001$.

REM, rapid eye movement; $\mathrm{SaO}_{2}$, oxygen saturation by pulse oximetry; TST, total sleep time.

oximetry had a specificity of $81 \%$ and a sensitivity of $87 \%$ for Cheyne-Stokes respiration.

NOCTURNAL DESATURATION

The baseline characteristics of the patients and normal controls are shown in tables 1 and 2 . Ninety one per cent of the subjects were male, and in $81 \%$ of cases the aetiology of heart failure was ischaemia. Twelve patients were excluded from study on the basis of known coexistent pulmonary disease or stroke. The results of overnight oximetry recordings are shown in table 3 . The mean (SD) total oximetry time for all subjects was 7 (1.2) hours. All indices of nocturnal desaturation were significantly greater among the patient group than in the age matched controls; the mean dip frequency (10.3/hour) was double that seen in the control group. Twenty three patients $(22 \%)$ had more than 15 dips/hour, compared with none in the control group, all of whom had a pattern of nocturnal desaturation categorised as "abnormal" by an independent observer (see fig 3 for specimen recording). "Abnormal" and "equivocal" traces were commoner in patients than in normal controls $\left(\chi^{2}=8.3 ; \mathrm{p}<0.05\right.$; fig 4) Only five patients had a mean $\mathrm{SaO}_{2}$ of less than $90 \%$ during sleep. There was no correlation between dip frequency and any of the known risk factors for obstructive sleep apnoea (age, obesity, and alcohol consumption). Ejection fraction was found to correlate negatively with dip frequency across the whole study group. $\log _{(10)}$ transformation of dip frequency was performed to produce a normal distribu-

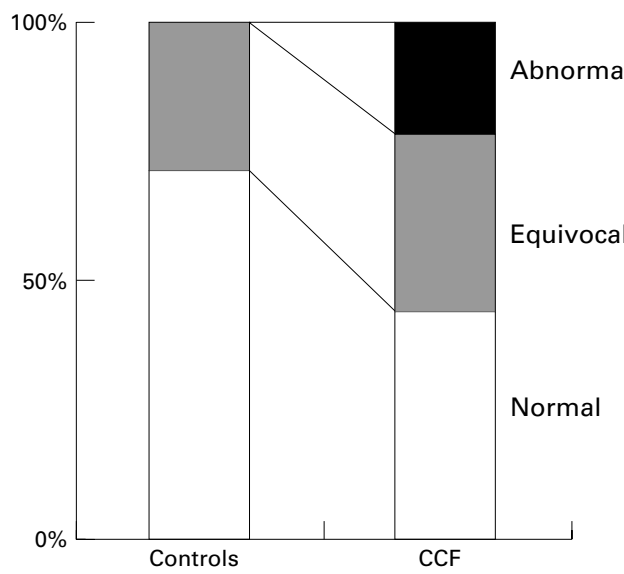

Figure 4 Cumulative histograms showing frequency of disordered breathing in 104 patients with chronic heart failure and 21 normal volunteers.

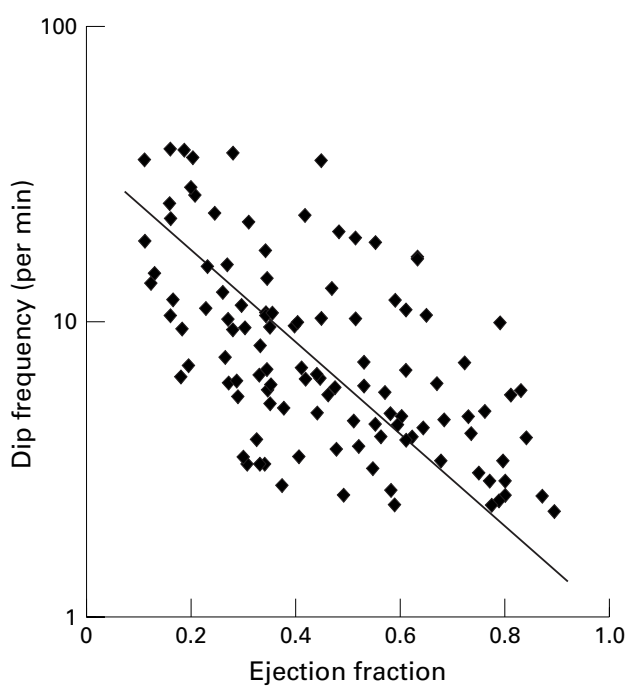

Figure 5 Relation between ejection fraction and desaturation events in all subjects.

tion before calculation of the Pearson correlation coefficient and simple linear regression to determine the line of best fit (fig $5 ; r=-0.5$; $\mathrm{p}<0.005)$. Analysis of the patient group in isolation showed similar results $(r=-0.45$, $\mathrm{p}<0.005$; not shown). This was to be expected since periodic respiration is seen even within a normal population, and ejection fraction must in part be related to circulatory delay which is important in the pathogenesis of Cheyne-Stokes respiration.

\section{POLYSOMNOGRAPH STUDY}

The results of polysomnography in a subgroup of 41 randomly selected patients with and without Cheyne-Stokes respiration are shown in table 4. Ninety six per cent of apnoeas in these patients with known heart failure were central in origin. Only $4 \%$ of apnoeas were obstructive, and no subject fulfilled the diagnostic criteria for obstructive sleep apnoea. As was to be expected, all indices of disordered breathing were significantly worse in that subgroup of patients with Cheyne-Stokes respiration (table 4).

NOCTURNAL ARRHYTHMIA

Patients with frequent dips also experienced significantly more ventricular extrasystoles and belonged to poorer NYHA functional classes (table 2). There was no relation between any marker of nocturnal desaturation and the severity of arrhythmia graded according to the Lown classification. None of our patients experienced episodes of sustained ventricular tachycardia during sleep.

\section{DAYTIME SLEEPINESS}

There was no correlation between any marker of nocturnal desaturation and assessment of daytime sleepiness using ESS scores. Thirty six patients had an ESS of more than 10, and there was a strong relation between NYHA functional class and symptoms of daytime sleepiness $\left(\chi^{2}=27.3, \mathrm{p}<0.005\right)$. 


\section{Discussion}

NOCTURNAL DESATURATION

The results of this study have shown that abnormal overnight oximetry is a common finding in patients with treated chronic heart failure. All the measured indices of nocturnal desaturation were greater in the patients than in the control subjects. Twenty three of the patients had "abnormal" oximetry recordings, subsequently shown to be typical of CheyneStokes respiration.

Sleep disordered breathing may be directly harmful to the cardiovascular system, or it may just disturb normal sleep architecture. Whatever the final result, the precise cause of the abnormalities is poorly understood. We have shown that it seems to be related-at least in part - to the degree of left ventricular dysfunction since there was a significant negative correlation between dip frequency and ejection fraction. For both theoretical and methodological reasons it was unsurprising that ejection fraction was responsible for only $25 \%$ of the variance in dip frequency in our study; although Cheyne-Stokes respiration is caused by impaired left ventricular function (circulatory delay), its severity is also related to "underdamping," increased "controller gain," and increases in the apnoeic threshold which we did not measure. Javaheri et al have shown a much stronger relation between ejection fraction and sleep disordered breathing using radionucleide ventriculography to measure ejection fraction and inpatient polysomnography. ${ }^{12}$ In a study similar to ours, Blackshear et al failed to show any relation between ejection fraction and desaturation index. ${ }^{13}$

In this paper we also present data on the validity and reproducibility of the use of home oximetry to screen for sleep disordered breathing in heart failure. It is obviously less precise than polysomnography (since it does not measure airflow or arousal) but has the advantage of being cheap and minimally disruptive, and it can easily be used in patients' own homes. The results from our polysomnography substudy show that the specificity and sensitivity of oximetry for detecting Cheyne-Stokes respiration are high. This suggests that overnight oximetry alone may be adequate to screen for Cheyne-Stokes respiration in the clinical setting of known heart failure without coexistent pulmonary disease.

NOCTURNAL ARRHYTHMIA

Previous studies have suggested that the presence of Cheyne-Stokes respiration is an independent predictor of death in patients with heart failure. ${ }^{5}$ One possible mechanism for this would be lethal arrhythmia associated with episodes of hypoxia. ${ }^{14}$ We investigated this by combining Holter monitoring with overnight oximetry. Patients with "frequent" nocturnal desaturations experienced significantly more ventricular ectopy, but there was no difference between the groups with respect to either Lown classification or the occurrence ventricular tachycardia. The results of this study do not support a direct link between episodes of Cheyne-Stokes respiration with nocturnal de- saturation and serious arrhythmia; the most probable reason for this is that although the frequency of desaturation events was increased the absolute level of desaturation observed was relatively mild. In these patients dip frequency told us more about the pattern of the disturbance in breathing during sleep than about the actual severity of nocturnal desaturation. It is also important to remember that none of our patients had unstable coronary syndromes. Furthermore, the prognostic significance of ventricular arrhythmia in cardiac failure remains unclear. Although half of all heart failure patients die suddenly, frequent ventricular ectopy and episodes of nonsustained ventricular tachycardia do not appear to act as good markers for sudden cardiac death. ${ }^{15}$

\section{DAYTIME SLEEPINESS}

Heart failure patients have more daytime somnolence than normal controls in our laboratory (unpublished data). Furthermore, this study has shown that those patients with poorer NYHA classification experienced more daytime sleepiness (ESS scores). ESS scores failed to correlate with any of the measured indices of nocturnal desaturation or ejection fraction. It would appear that general symptoms are linked to daytime sleepiness, but this is unrelated to either the frequency or severity of nocturnal desaturation - probably because oximetry cannot measure arousal from sleep.

\section{STUDY LIMITATIONS}

Our validation study of polysomnography versus oximetry was performed in the hospital environment. Unfortunately we did not have access to polysomnography equipment that can be installed in patients' own homes, and ideally this should have been used.

Ejection fraction in our study was quantified by $M$ mode echocardiography. This technique is less reliable in patients with regional left ventricular dysfunction than in those with global impairment. This may in part explain the better correlation between ejection fraction and disordered breathing observed by other investigators, ${ }^{12}$ and the apparently well preserved ejection fractions in some of our patients.

\section{CONCLUSIONS}

We have shown that episodes of nocturnal desaturation are common in patients with stable heart failure receiving optimal medical treatment, and that dip frequency is inversely related to ejection fraction. Serious ventricular arrhythmia was not observed. Although oximetry cannot distinguish central from obstructive apnoeas, the results of our polysomnography substudy suggests that in the clinical setting of known heart failure oximetry alone is an adequate screening test for Cheyne-Stokes respiration. This is important because CheyneStokes respiration is an independent predictor of death in heart failure. Daytime somnolence was unrelated to nocturnal desaturation. 
1 Cheyne J. A case of apoplexy in which the fleshy part of the heart was converted into fat. Dublin Hospital Reports

2 Findley LJ, Zwillich CW, Ancoli-Israel S, et al. CheyneStokes breathing during sleep in patients with left ventricular failure. South Med f 1985;78:11-15.

3 Ancoli-Israel S, Engler RL, Friedman PJ, et al. Comparison of patients with central sleep apnoea-with and without Cheyne-Stokes respiration. Chest 1994;106:780-6.

4 Hanly PJ, Millar TW, Steljes DG, et al. Respiration and abnormal sleep in patients with congestive heart failure. Chest 1989;96:480-8.

5 Hanly PJ, Zuberi-Khokhar NS. Increased mortality associated with Cheyne-Stokes respiration in patients with congestive heart failure Am $f$ Resp Crit Care Med 1996;153:272-6.

6 Johns MW. A new method for measuring daytime sleepiness: the Epworth sleepiness scale. Sleep 1991;14: sleepiness: the Epworth sleepiness scale. Sleep 1991;14:
540-5.

7 Johns MW. Daytime sleepiness, snoring and obstructive sleep apnoea. The Epworth sleepiness scale. Chest 1993; 103:30-6.

8 Warley ARH, Stradling JR, Mitchell J. Evaluation of Ohmeda 3700 pulse oximeter. Thorax 1987;42:892-6.
9 Stradling JR, Crosby JH. Predictors and prevalence of obstructive sleep apnoea and snoring in 1001 middle aged obstructive sleep apnoea and
men. Thorax 1991;46:85-90.

10 Rechtschaffen A, Kales A. A manual of standardized terminology, techniques, and scoring system for sleep stages of human subjects. Publication No 204. Washington DC: National Institutes of Health.

11 Hanly PJ, Millar TW, Steljes DG, et al. The effect of oxygen on respiration and sleep in patients with congestive heart on respiration and sleep in patients with

12 Javaheri S, Parker TJ, Wexler L, et al. Occult sleep disordered breathing in stable congestive heart failure. Ann Intern Med 1995;122:487-92.

13 Blackshear JL, Kaplan J, Thompson RC, et al. Nocturnal dyspnoea and atrial fibrillation predicts Cheyne-Stokes respiration in patients with congestive heart failure. Arch Intern Med 1995;155:1297-302.

14 Cripps T, Rocker G, Stradling J. Nocturnal hypoxia and arrhythmias in patients with impaired left ventricular funcarronythr Br Heart f 1992;68:382-6.

15 Packer M. Sudden unexpected death in patients with congestive heart failure: a second frontier. Circulation 1985; 72:681-5 